\title{
Creation of Three-Dimensional Micro-Photonic Structures on the End-Face of Optical Fibers
}

\author{
Stephen. M. KUEBLER ${ }^{* 1,2,3}$, Henry E. WILLIAMS ${ }^{1}$, Daniel J. FREPPON ${ }^{1}$, \\ Raymond C. RUMPF ${ }^{4}$, Marco A. MELINO ${ }^{2}$ \\ ${ }^{1}$ Chemistry Department, University of Central Florida, Orlando, FL 32816 \\ ${ }^{2}$ CREOL, The College of Optics and Photonics, University of Central Florida, Orlando, FL 32816 \\ ${ }^{3}$ Physics Department, University of Central Florida, Orlando, FL 32816 \\ E-mail: stephen.kuebler@ucf.edu \\ ${ }^{4}$ EM Lab, W. M. Keck Center for 3D Innovation, The University of Texas at El Paso, \\ El Paso, TX 79968
}

\begin{abstract}
A process is reported that enables fabrication of truly three-dimensional micro-photonic structures directly onto the end face of an optical fiber by multi-photon direct laser writing in the cross-linkable epoxide SU-8. Solvent-free SU-8 resin is first obtained by heating in vacuo to remove volatiles. The resulting resin solids are then melt-reflowed around an optical fiber in a mold integrated into a sample mount. The resin is allowed to cool and solidify around the optical fiber, so the entire sample mount can be affixed to an optical system for direct laser writing. Using this approach a wide range of refractive and diffractive micro-optical structures can be integrated onto optical fibers that would be difficult, if not impossible, to create by other existing methods. Optical characterization of lens-tipped fibers shows that the approach can be used control the propagation of beams exiting from functionalized fibers, and the performance is reproducible across repeated fabrication of the same device. This work illustrates a new path to fiber-based integrated photonic devices.
\end{abstract}

DOI:10.2961/jlmn.2012.03.0011

Keywords: Nanophotonics; Microstructure fabrication; Photonic crystals; Laser materials processing; Micro-optics.

\section{Introduction}

Integrated micro-optical devices are increasingly sought as solutions to challenges in sensing, telecommunications, and optical-interconnects for inter- and intra-microchip data transfer [1]. Optical fibers remain among the most widely used platforms for integrated optical devices. They can be manufactured inexpensively and to high performance tolerances in glasses and polymers, and a wider range of materials that is expanding to include chalcogenides and semiconductors [2,3]. Yet, creating integrated photonic devices using fibers remains challenging. At the same time, multi-photon direct laser writing (DLW) is advancing rapidly and establishing itself as among the most powerful and flexible methods for creating truly three-dimensional micro- and nano-scale structures in polymers, and other materials [4, 5]. DLW has been used to create a wide range of refractive and diffractive micro-optical devices $[6,7]$, and the technique has been used to integrate such structures directly onto optical fibers using liquid photo-polymer resins [8-11].

Here we describe an approach we developed for processing the cross-linkable epoxide SU-8 and then using it to fabricate micro-optical elements directly onto the endface of optical fibers. SU-8 was selected because the material is a high-performance, low-shrinkage material [12] that is increasingly applied for creating micro-electromechanical systems (MEMS) and optical
MEMS (OMEMS) [13, 14]. Additionally, it offers high thermal and chemical resistance relative to other crosslinkable media [15], so integrated micro-optical devices created with it could be suitable for applications in extreme conditions [16].

\section{Experimental}

\subsection{Isolation of $\mathrm{SU}-8$ resin solids}

SU-8 2075 is supplied commercially (MicroChem Corporation) as a clear viscous liquid consisting of SU-8 (an epoxide-functionalized bisphenol A oligomer, CAS 2890696-9, $70 \mathrm{wt}-\%$ ), two triarylsulfonium hexafluoroantimonate photoacid generators (CAS 89452-37-9 and 71449-78-0, 3.5 wt- $\%$ total), cyclopentanone solvent (CAS 120-92-3), and propylene carbonate (CAS 108-32-7), which functions as a plasticizer. A round bottom flask was loaded with a magnetic stir bar and portions of SU-8 2075 (typically $\sim 10 \mathrm{~mL}$ ) then affixed to a rotary evaporator and immersed in an oil bath heated to $95^{\circ} \mathrm{C}$. Vacuum was applied gradually to reduce pressure, causing the solvent to vaporize. This process was continued to remove the majority of the volatiles. The round bottom flask was then connected to a vacuum line and heated for various periods with full vacuum applied $(<0.5$ Torr) to remove remaining solvent. The resin was the removed from vacuum and while still molten it was poured onto a glass plate and allowed to solidify. 
The resulting solids were broken into small pieces and stored for later use. Proton nuclear magnetic resonance spectroscopy ( ${ }^{1} \mathrm{H} \mathrm{NMR}$ ) was used to quantify the solvent content of the solidified resin by comparing the ratio of integrated areas for peaks corresponding to the solvent with those due to SU-8 resin and comparing against those measured for the resin as supplied. The solvent content is reported as a weight-percent.

\subsection{Sample preparation}

A mount was constructed (Fig. 1) for immersing an optical fiber into a plug of vacuum-baked SU-8 resin at controlled depth. The mount consists of a fiber chuck affixed to a micrometer-controlled translation stage supported by an $L$-bracket above a clear-bored aluminum plate. The aluminum plate serves as a mold for casting vacuum-baked resin around the fiber.
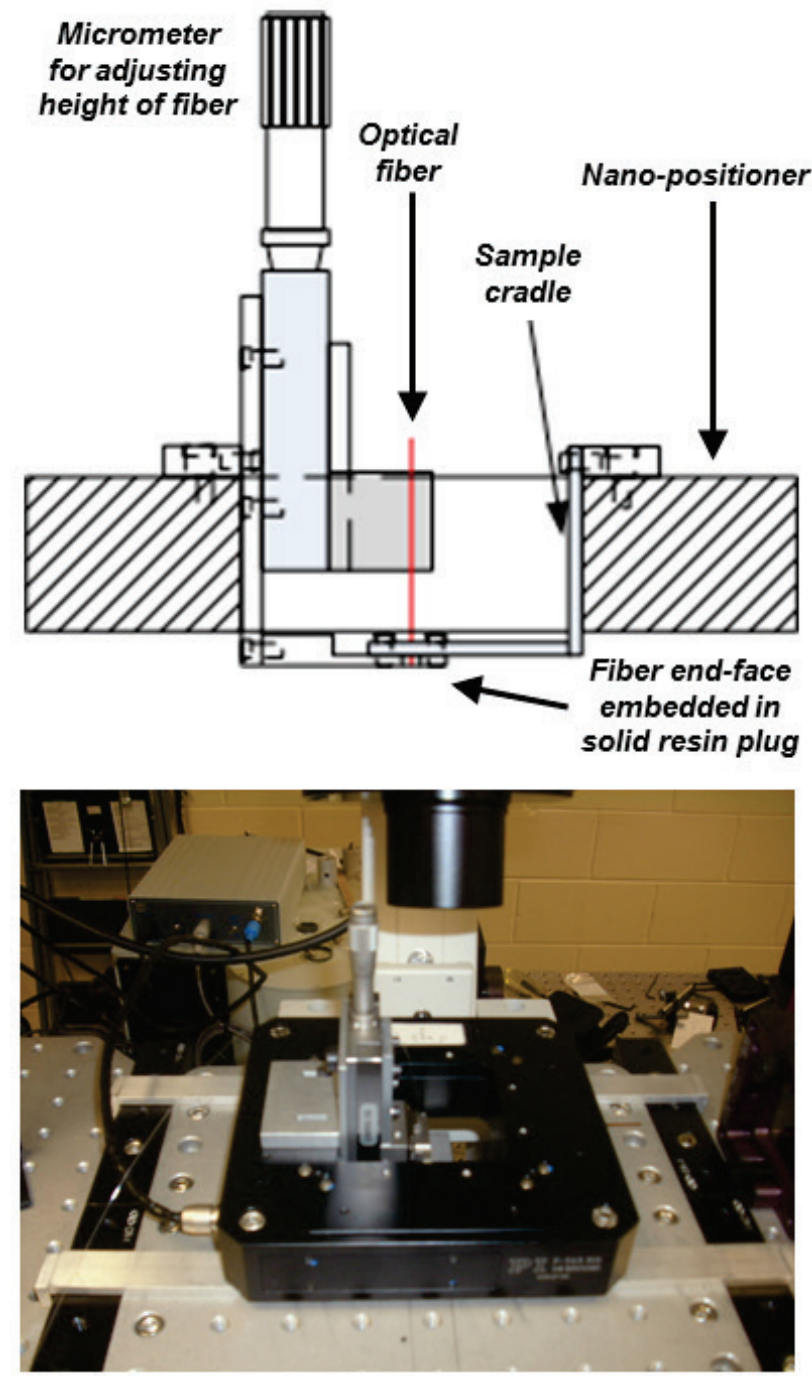

Fig. 1 (Top) Schematic of the sample holder used for multiphoton direct laser writing of structures onto the end-face of an optical fiber. (Bottom) Photograph of the optical-fiber sample holder mounted onto a three-axis nano-positioner for multiphoton patterning of the micro-structure.

Micro-optical structures were fabricated onto singleand multi-mode optical fibers (Nufern/ThorLabs $630 \mathrm{HP}$ and 1060XP, $\lambda=633 \mathrm{~nm}$ ). All fibers were hand-cleaved. A 1-cm length of the plastic jacket was removed from each end of the fiber. The exposed cladding was scored with a diamond-tipped knife then bent to initiate the cleave. The freshly cleaved fiber was then affixed to the mount and the micrometer was rotated to insert the fiber into the resin mold until a desired spacing remained between the end face and the bottom surface of the mold (typically $50 \mu \mathrm{m}$ ). A microscope coverslip was placed in contact with the bottom of the mold and the entire unit was transferred to a hot plate heated to $110^{\circ} \mathrm{C}$. Pieces of vacuum-baked SU-8 were inserted into the mold and allowed to melt and re-flow around the fiber tip. Once all bubbles had escaped from the melt, the hot plate was switched off and the mount was allowed to cool. When the mount reached room temperature, slight pressure was applied to the cover glass causing it to release from the resin plug. The resulting assembly held an optical fiber embedded to controlled depth in a low-solvent-content 1- to 2-mm thick plug of SU-8 resin, ready for fabrication of the micro-structure onto the fiber end-face.

\subsection{Direct laser writing}

The optical configuration used for DLW of microoptics onto the end-face of optical fibers consists of writing-, imaging-, and illumination sub-systems. The writing and imaging sub-systems are similar to those reported previously for conventional multi-photon DLW [4]. The fiber mount is affixed to a three-axis nanopositioner (Physik Instrumente P-563.3CD) supported above the objective of an inverted microscope (Nikon TE2000-U). The output of a continuous-wave modelocked femtosecond laser at a center wavelength of $800 \mathrm{~nm}$ (Coherent-Mira, 120-fs pulse duration, $76 \mathrm{MHz}$ repetition rate) is routed through a shutter, a half-wave plate/polarizer combination, a beam expander, and then into a $100 \times / 1.4 \mathrm{NA}$ oil-immersion objective (Nikon) that focuses the laser pulses into the resin plug from underneath the sample holder, seen in Fig. 1. The average focused power used was $2.4-3.0 \mathrm{~mW}$, as measured at the exit aperture of the objective.

The illumination sub-system provides a convenient means for locating the core of the fiber relative to the writing beam in both the transverse and axial directions. $\mathrm{HeNe}$ laser light coupled into the free end of the fiber emerges from the end-face immersed in the resin plug, enabling the core to be imaged with a CCD camera. The fiber mount is then translated until the reflected image of the write beam is coincident with the illuminated core, providing a reference point for fabrication of the target structure. This approach enables us to position the microstructure with respect to the fiber core.

Micro-optics were patterned under computer control, driven by a fabrication path plan. The path plan consists of a grid of line segments along which the laser beam is tracked to define the internal volume of the structure. The spacing of lines is selected to ensure that the features generated at a given laser power will overlap and fuse forming a continuous cross-linked internal structure. The processing conditions described here generate polymerized lines having a lateral width of circa $0.5 \mu \mathrm{m}$. The entire 
sample mount, and thus the end-face of the fiber, is translated relative to the focal spot at $50 \mu \mathrm{m} / \mathrm{s}$, as the shutter, attenuator, and nanopositioner are synchronously controlled from the microcomputer. Following exposure, samples are baked to activate cross-linking (15 min. at hotplate temperature of $105^{\circ} \mathrm{C}$ ), transferred to a glass plate and allowed to cool (5 min), immersed in propylene glycol methyl ether acetate to remove unexposed resin, rinsed with methanol, then dried in air, leaving a free-standing micro-structure remaining on the substrate.

\subsection{Structure characterization}

Scanning electron microscopy (SEM) was used to characterize the three-dimensional form and integrity of microstructures fabricated on the end-face of an optical fiber and to obtain quantitative information about their dimensions. A HeNe laser was coupled into the unfunctionalized end of the fiber and a laser beam profiler was used to image the pattern of beams emerging from the end bearing a microstructure. For lens-tipped fibers, the patterns analyzed well as Guassian spatial profiles, for which the spot size could be characterized in terms of the full-width at half-maximum (FWHM) obtained from a line scan through the beam profile. Irradiance patterns formed by lens-tipped fibers were imaged as a function of distance from the end face to obtain the beam divergence for correlation with the radius of curvature of the lens.

\section{Results and Discussion}

Figures 2 and 3 show examples of a plano-convex lens created by DLW on the end face of a single-mode optical fiber. This particular lens was fabricated to have a targeted radius of curvature of $R=20 \mu \mathrm{m}$. From SEM images, the actual curvature was found to be $R=19.0 \mu \mathrm{m}$. Other plano-convex lenses were created having targeted (observed) values of $R$ equal to $40 \mu \mathrm{m}$ (34.1 $\mu \mathrm{m}$ found) and $20 \mu \mathrm{m}(18.3 \mu \mathrm{m}$ found). Close inspection of Fig. 2 reveals that the lens is well formed and the surface is smooth. Fig. 3 shows the same lens looking down onto the end-face of the optical fiber. The lens is well formed, rotationally symmetric, and well centered with respect to the fiber and its core. The optical characterization of these micro-lenses was reported elsewhere [16]. The far field patterns formed by the HeNe beam coupled into this fiber formed a uniformly Gaussian spatial irradiance distribution in the far field that diverged faster than that emerging from an unfunctionalized fiber. Additionally, the divergence angle was found to increase as the radius of curvature of a given micro-lens decreased, and thus the focusing power increased. This behavior is consistent with the beam being focused by the micro-lens near the end-face. In all cases, the far-field patterns were highly uniform, speckle-free Gaussian distributions, which is as expected given the smooth surface of the micro-lens.

Comparison of the targeted and measured radii of curvature indicates that the final structures deviate from the planned form by as much as $10-15 \%$. This results most likely from re-flow that occurs during the post-exposure bake step. Evidence for this can be seen in the lower panel of Fig. 2, where the side walls are sloped inward, and the vertical lines used to define the internal volume of the lens are visible as "fringes" along the outer perimeter. As discussed below, further refinement of the processing conditions, such as bake time and bake temperature, could lead to improvement in the fidelity of the structures. It is noteworthy, however, that the beam divergence angles were found to be nearly indistinguishable for repeated fabrication of the same device, suggesting the approach is repeatable.

"Woodpile" 3D diffractive structures were fabricated onto the end-face of optical fibers to illustrate the potential of the method as a means for creating more complex integrated photonic devices. Figure 4 is an optical transmission image in side-view of a face-centered tetragonal woodpile structure that was fabricated on the end-face of a multi-mode optical fiber. The structure consists of parallel rods with a center-to-center spacing of $2.5 \mu \mathrm{m}$ and stacked in offset layers spaced by $0.9 \mu \mathrm{m}$ [17]. Five layers complete a square array of unit cells. Structures having several different numbers of layers were prepared. Figure 5A shows an SEM image of a woodpile structure on the end-face of a multi-mode fiber and fabricated with " $L$-shaped" anchors at the corners. The anchors help reinforce the structure and improve its adhesion to the surface by increasing the contact area.

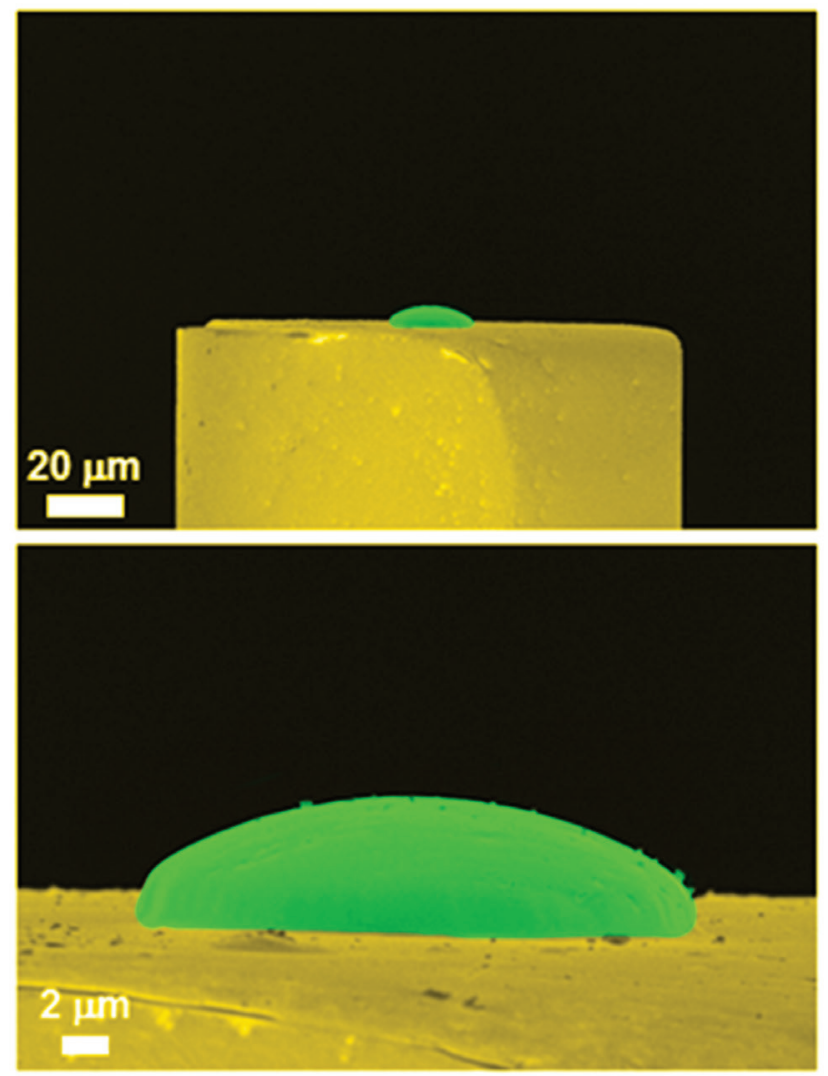

Fig. 2 False-color SEM images of a plano-convex lens fabricated onto the end-face of an optical fiber. The lens was designed to have a radius of curvature of $R=20 \mu \mathrm{m}$.

Figures $5 \mathrm{~B}$ and $5 \mathrm{C}$ enable us to examine the effect the woodpile structure shown in Fig. 5A has on the output of the fiber. From Fig. 5B we see that $\mathrm{HeNe}$ laser light coupled into a bare fiber exits generating two lobes in the far field, consistent with the multi-mode character of the fiber. In contrast, Fig. 5C shows that the same type of multi-mode fiber bearing a woodpile structure on its end- 
face generates a complex multi-spot pattern in the far field. Separate experiments involving a woodpile structure fabricated on a simple glass substrate and probed using gently focused HeNe laser beam suggest that the far field pattern generated by a fiber bearing the structure is complex mix of diffraction spots and scatter. The scatter most likely results because the lateral dimension of the fiber core is comparable to the rod spacing, so the emerging beam samples few parallel rods comprising the woodpile structure and is strongly affected by local structural variation in the few rods with which it interacts upon traversing the $3 \mathrm{D}$ structure.

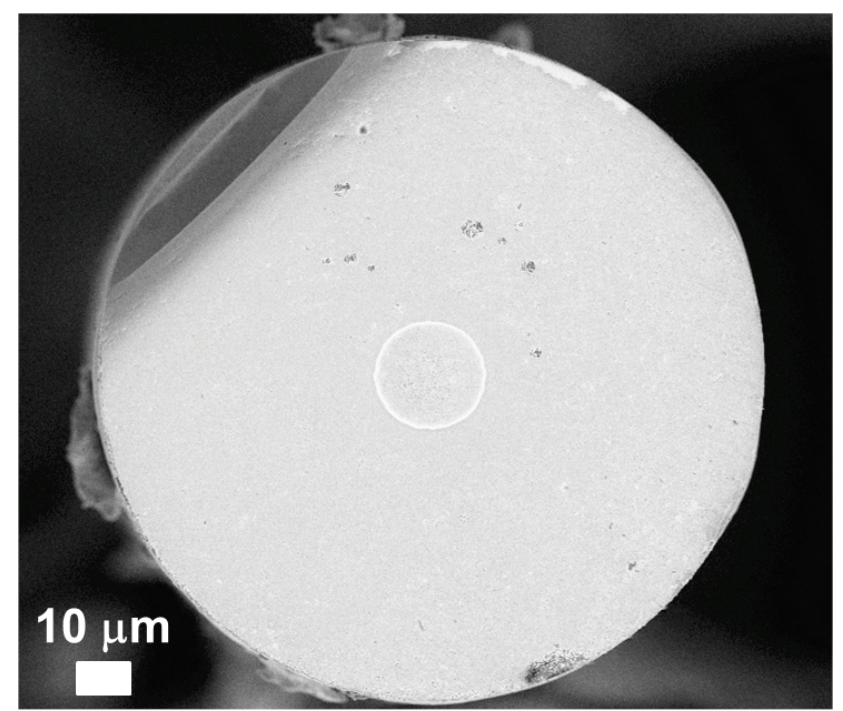

Fig. 3 SEM image of the plano-convex lens looking down onto the end-face of the optical fiber onto which it was fabricated.

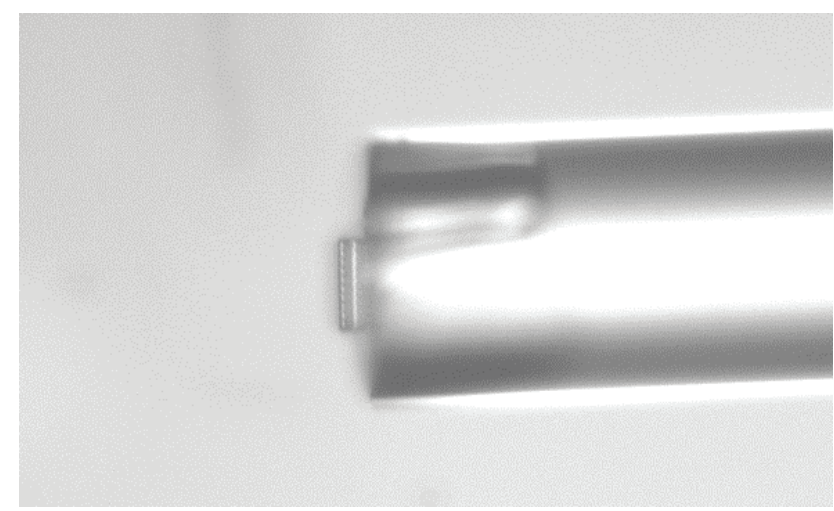

Fig. 4 Optical image of a face-centered tetragonal woodpile structure fabricated onto the end-face of an optical fiber by multiphoton direct laser writing.

It is well known that the quality and integrity of microstructures produced using SU-8 depend critically on DLW exposure conditions as well as those used prior and following exposure [18-20]. This is particularly true in the present case where the additional steps of removing solvent in vacuo and melt-reflow in the mold are used to enable DLW on the end-face of the optical fiber. The complex space associated with material processing was explored only in the most superficial sense to enable fabrication of the micro-structures shown here, but evidence is obtained that the additional processing steps have a critical effect on the micro-structure quality, and there remains considerable room for further improvement and fundamental study.
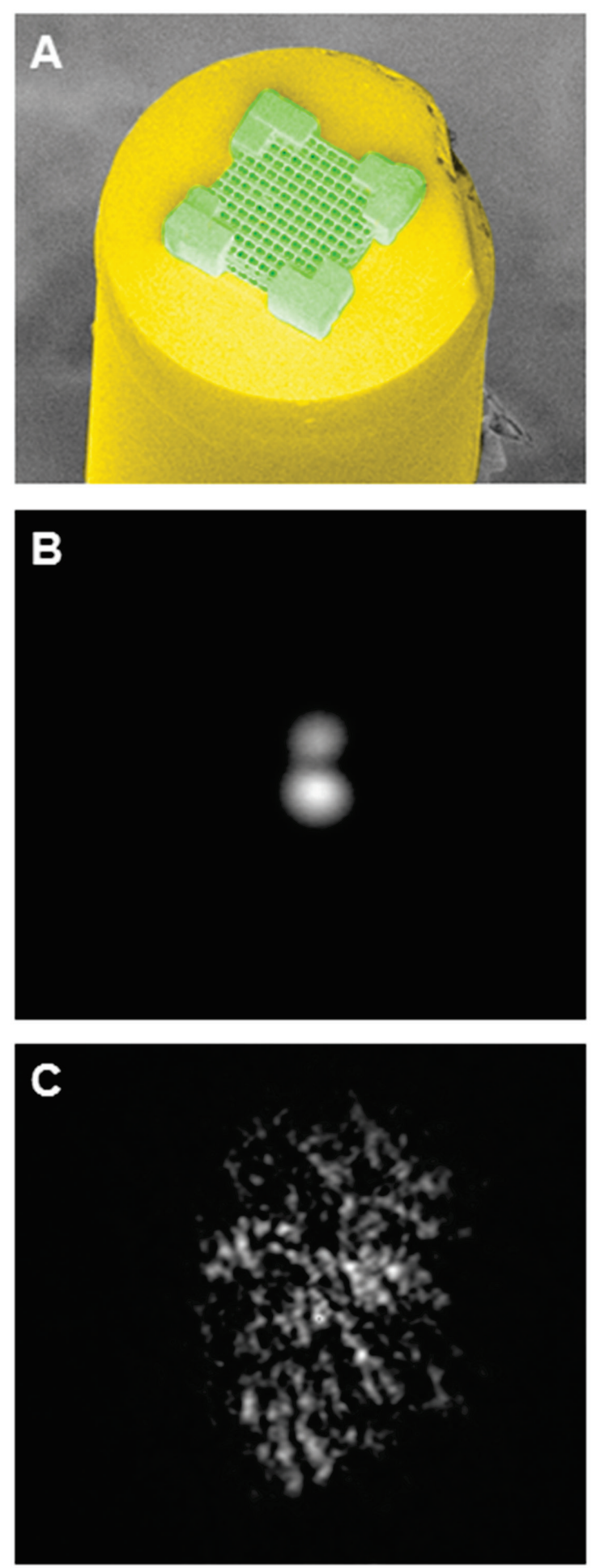

Fig. 5 (Top) False-color SEM image of a face-centered tetragonal 3D woodpile structure fabricated onto the end-face of a multimode optical fiber by multi-photon direct laser writing. (Middle \& Bottom) Irradiance profiles of the beam emerging from a (middle) bare multi-mode optical fiber and (bottom) one supporting a face-centered tetragonal woodpile structure.

Figure 6 is a composite of SEM images taken as topand side-views of woodpile structures created by DLW 
using SU-8 resin that was processed with different lengths of pre-exposure baking, so as to have varying amounts of residual solvent. It should be noted that the increase in sharpness that exists between images along the progression from highest to lowest solvent content is not a result of poor imaging, but rather due to the quality and form of the polymeric microstructures themselves. Each structure was prepared using resin with comparatively low solvent content ( $\sim 1 \mathrm{wt}-\%$ or less), yet the images show clearly that even small amounts of residual solvent compromise the structure quality. Those created with $\sim 1 \mathrm{wt}-\%$ residual solvent have irregular features that vary across the structure, and the interior of the lattice is ill defined, as though significant diffusion and resin re-flow occurs during the post-exposure bake step used to initiate cross-linking. As the residual solvent content is reduced, the microfabricated features become more sharply defined. The woodpile structure prepared with $0.68 \mathrm{wt}-\%$ solvent (14 hours of preexposure baking) is well formed, and the features are far more regular across the surface.

\section{Solvent wt-\% = 1.07}

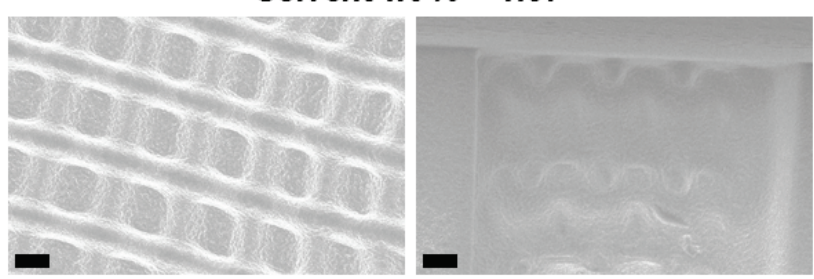

\section{Solvent wt- $\%=0.98$}
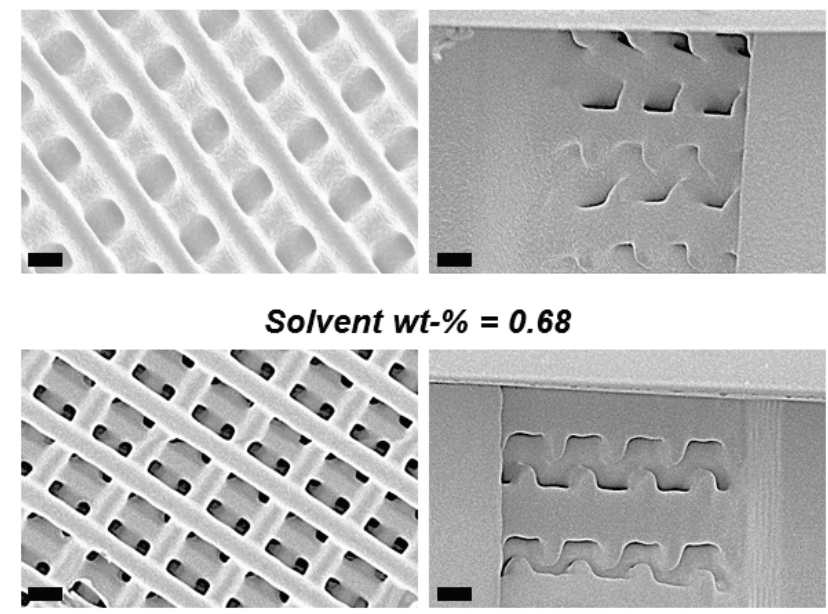

Fig. 6 SEM images of face-centered tetragonal 3D woodpile structures fabricated on the end-face of an optical fiber using SU8 resin having different amounts of residual solvent, as viewed from the top (left column) and side (right column). All scale bars correspond to a length of $2 \mu \mathrm{m}$.

We did not attempt to improve the structure fidelity further by removing additional solvent because baking the resin for more than 14 hours was regarded as impractical. However, these results suggest that a further exploration of the material processing conditions, such as optimizing the amount of residual solvent, bake temperatures, and pre- and post-exposure bake times, could further improve the quality of integrated micro-devices that can be prepared using this approach and their fidelity relative to targeted design parameters $[19,20]$.

\section{Conclusion}

This work describes a method for preparing integrated photonic devices in which micro-structures are fabricated directly on the end-face of single- and multi-mode optical fibers and provides examples of the types of refractive and diffractive three-dimensional micro-optics that can be prepared using this approach. The fabrication method involves an adaptation of multi-photon DLW using the high performance cross-linkable epoxide resin, SU-8. Solvent was removed from the resin by heating in vacuo prior to exposure so the remaining SU-8 solids could be meltreflowed into a mold around the optical fiber. This approach provides a stable integrated sample for DLW that eliminates the need to spin-coat SU-8, which is impractical when fabricating onto optical fibers. Preliminary optical characterization suggests the approach can be used to create integrated fiber-based devices with useful optical function, opening a route to new route to novel fiber-based devices.

\section{Acknowledgments}

This work was supported by NSF CAREER grant 0748712 . D. J. Freppon has been partially supported by NSF grants 0525429 and 0806931 . The authors thank the referees for their helpful comments.

\section{References}

[1] G. Lifante, Integrated Photonics: Fundamentals (John Wiley \& Sons, Chichester, UK, 2005).

[2] A. F. Abouraddy, O. Shapira, M. Bayindir, J. Arnold, F. Sorin, D. S. Hinczewski, J. D. Joannopoulos, and Y. Fink, "Large-scale optical-field measurements with geometric fibre constructs," Nature Mater. 5, 532-536 (2006).

[3] J. Ballato, T. Hawkins, P. Foy, R. Stolen, and R. Rice, "Advancements in semiconductor core optical fiber," Opt. Fiber Technol. 16, 399-408 (2010).

[4] S. M. Kuebler and M. Rumi, "Nonlinear optics -applications: three-dimensional microfabrication," in Encyclopedia of Modern Optics, R. D. Guenther, D. G. Steel, and L. Bayvel, eds. (Elsevier, Oxford, 2004), pp. 189-206.

[5] L. Li and J. T. Fourkas, "Multiphoton polymerization," Mater. Today 10(6), 30-37 (2007).

[6] R. Guo, S. Xiao, X. Zhai, J. Li, A. Xia, and W. Huang, "Micro lens fabrication by means of femtosecond two photon photopolymerization," Opt. Express 14(2), 810-816 (2006).

[7] M. Malinauskas, A. Žukauskas, K. Belazaras, K. Tikuišis, V. Purlys, R. Gadonas, and A. Piskarskas, "Laser fabrication of various polymer microoptical components," Eur. Phys. J. Appl. Phys. 58, 2050120501 - 20501-20508 (2012).

[8] T. Sherwood, A. C. Young, J. Takayesu, A. K. Y. Jen, L. R. R. Dalton, and A. Chen, "Microring resonators on side-polished optical fiber," IEEE Photon. Technol. Lett. 17(10), 2107-2109 (2005).

[9] G. Cojoc, C. Liberale, P. Candeloro, F. Gentile, G. Das, F. De Angelis, and E. Di Fabrizio, "Optical microstructures fabricated on top of optical fibers by means 
of two-photon photopolymerization," Microelectron. Eng. 87, 876-879 (2010).

[10] M. Malinauskas, A. Žukauskas, V. Purlys, K. Belazaras, A. Momot, D. Paipulas, R. Gadonas, A. Piskarskas, H. Gilbergs, A. Gaidukevičiūtè, I. Sakellari, M. Farsari, and S. Juodkazis, "Femtosecond laser polymerization of hybrid/integrated microoptical elements and their characterization," J. Opt. 12, 1274040-1274041 - 1274040-1274048 (2010).

[11] M. Malinauskas, A. Žukauskas, V. Purlys, A. Gaidukevičiūtè, Z. Balevičius, A. Piskarskas, C. Fotakis, S. Pissadakis, D. Gray, R. Gadonas, M. Vamvakaki, and M.Farsari, "3D microoptical elements formed in a photostructurable germanium silicate by direct laser writing," Opt. Lasers Eng. 50, 1785-1788 (2012).

[12] L. J. Guerin, M. Bossel, M. Demierre, S. Calmes, and P. Renaud, "Simple and low cost fabrication of embedded microchannels by using a new thick-film photoplastic," presented at the Proceedings of the International Solid State Sensors and Actuators Conference (Transducers '97), Chicago, 16-19 June 1997, 1997.

[13] H. Lorenz, M. Despont, N. Fahrni, N. LaBianca, P. Renaud, and P. Vettiger, "SU-8: a low-cost negative resist for MEMS," J. Micromech. Microeng. 7, 121124 (1997).

[14] K. Y. Lee, N. LaBianca, S. A. Rishton, S. Zolgharnain, J. D. Gelorme, J. Shaw, and T. H.-P. Chang, "Micromachining applications of a high resolution ultrathick photoresist," J. Vac. Sci. Technol. B 13(6), 3012-3016 (1995).

[15] N. C. LaBianca and J. D. Gelorme, "High aspect ratio resist for thick film applications," Proc. SPIE 2438, 846-852 (1995).

[16] H. E. Williams, D. J. Freppon, S. M. Kuebler, R. C. Rumpf, and M. A. Melino, "Fabrication of threedimensional micro-photonic structures on the tip of optical fibers using SU-8," Opt. Express 19(23), 22910 - 22922 (2011).

[17] A. Tal, Y.-S. Chen, H. E. Williams, R. C. Rumpf, and S. M. Kuebler, "Fabrication and characterization of three-dimensional copper metallodielectric photonic crystals," Opt. Express 15(26), 18283-18293 (2007).

[18] K. K. Seet, J. Juodkazytè, V. Jarutis, and H. Misawa, "Feature-size reduction of photopolymerized structures by femtosecond optical curing of SU-8," Appl. Phys. Lett. 89, 024106-024101 - 024106024103 (2006).

[19] R. G. Denning, C. F. Blanford, H. Urban, H. Bharaj, D. N. Sharp, and A. J. Turberfield, "The control of shrinkage and thermal instability in SU-8 photoresists for holographic lithography," Adv. Funct. Mater. 21(9), 1593-1601 (2011).

[20] J. Zhang, K. L. Tan, G. D. Hong, L. J. Yang, and H. Q. Gong, "Polymerization optimization of SU-8 photoresist and its applications in microfluidic systems and MEMS," J. Micromech. Microeng. 11, 20-26 (2001).

(Received: July 18, 2012, Accepted: September 18, 2012) 